\title{
Gérard Duménil, Dominique Lévy, La grande bifurcation. En finir avec le néolibéralisme
}

Paris, La Découverte, coll. «L'horizon des possibles », 2014

\section{Thomas Coutrot}

\section{OpenEdition}

Journals

Édition électronique

URL : http://journals.openedition.org/travailemploi/6660

DOI : $10.4000 /$ travailemploi.6660

ISSN : 1775-416X

Éditeur

DARES - Ministère du Travail

Édition imprimée

Date de publication : 1 avril 2015

Pagination : 99-101

ISSN : 0224-4365

Référence électronique

Thomas Coutrot, « Gérard Duménil, Dominique Lévy, La grande bifurcation. En finir avec le néolibéralisme », Travail et Emploi [En ligne], 142 | avril-juin 2015, mis en ligne le 01 avril 2015, consulté le 22

septembre 2020. URL : http://journals.openedition.org/travailemploi/6660 ; DOI : https://doi.org/

$10.4000 /$ travailemploi.6660

Ce document a été généré automatiquement le 22 septembre 2020.

(c) Direction de l'animation de la recherche, des études et des statistiques (Dares) 


\section{Gérard Duménil, Dominique Lévy, La grande bifurcation. En finir avec le néolibéralisme}

Paris, La Découverte, coll. « L'horizon des possibles », 2014

\section{Thomas Coutrot}

\section{RÉFÉRENCE}

Gérard Duménil, Dominique Lévy, La grande bifurcation. En finir avec le néolibéralisme,

Paris, La Découverte, coll. « L'horizon des possibles », 2014, 199 p.

1 Gérard Duménil et Dominique Lévy ont apporté depuis vingt ans des contributions majeures et mondialement reconnues à la compréhension du néolibéralisme. Fidèles à la méthode marxiste, ils placent la lutte des classes au cœur de la dynamique économique. Pour eux, le néolibéralisme n'est pas une idéologie mais une alliance de classes, entre les capitalistes et les cadres, sous l'hégémonie des premiers. La crise de 2008 a montré que ce dispositif était entré dans une phase de profondes perturbations qui pourraient mener à son éclatement. La grande bifurcation tente d'éclairer les devenirs possibles du capitalisme en s'appuyant à la fois sur une synthèse passionnante de riches travaux empiriques et sur un cadre théorique original.

2 À la structure de classes duale décrite par Marx, Duménil et Lévy proposent en effet de substituer une structure ternaire. Outre les prolétaires (qui, selon la définition classique, sont contraints pour vivre de vendre leur force de travail) et les capitalistes (propriétaires des moyens de production), une troisième classe, celle des cadres, gère les moyens de production pour le compte des propriétaires, grâce aux compétences de ses membres. Les cadres, selon Duménil et Lévy, recouvrent une définition plus étroite que la définition statutaire des cadres en France : il s'agit pour l'essentiel des dirigeants et financiers, soit environ $5 \%$ du salariat au lieu d'un tiers. 
3 Cette analyse prolonge des travaux déjà classiques sur les managers et la technostructure (James Burnham et la « révolution managériale ${ }^{1}$ ", John K. Galbraith et la «technostructure ${ }^{2}$ ", Alfred D. Chandler et la " main visible des managers ${ }^{3}$ " mais aussi Pierre Bourdieu et la dualité «capital culturel (notamment scolaire)»/«capital économique ${ }^{4}$ ", etc.). Le " socialisme réel » soviétique est ainsi réinterprété par Duménil et Lévy comme manifestant la domination des cadres. L'intérêt de cette structure ternaire est de sortir de l'alternative entre capitalisme et socialisme, en introduisant de possibles régimes intermédiaires. Le projet politique défendu par les auteurs est alors la dissolution de l'alliance entre les capitalistes et les cadres, et la recherche d'un compromis à gauche entre les cadres et les classes populaires.

Cette innovation théorique intéressante pose néanmoins question. Peut-on définir une classe uniquement par la position d'un groupe social dans les rapports de production? Les classes existent-elles "objectivement», indépendamment de leur capacité à se constituer en acteur collectif, doté d'une vision et d'une conscience des intérêts communs à ses membres ? Quel est l'intérêt analytique et politique de distinguer une "classe en soi » qui ne serait pas aussi une "classe pour soi », pour reprendre la distinction de Marx? Duménil et Lévy nous disent peu de chose de la conscience de classe des cadres, de leurs organisations ou de leur stratégie.

Mais surtout, les cadres dirigeants qui bénéficient de « salaires » extrêmement élevés et de stock options, ne détiennent-ils pas tous des patrimoines financiers considérables, qui les rapprochent fortement de la classe capitaliste? Il est vrai que selon l'enquête Patrimoine de l'Insee ${ }^{5}$, en 2010, un tiers du patrimoine des $1 \%$ les plus riches consiste en actifs d'entreprises, délimitant clairement une classe de propriétaires directs des moyens de production (cette proportion ne dépasse guère $10 \%$ pour les autres riches, un peu moins riches, du dixième décile). Cependant, le patrimoine financier liquide (au moins celui déclaré à l'Insee) représente aux alentours de $20 \%$ du patrimoine de tous les riches du dixième décile, qu'on les considère comme capitalistes ou cadres. Certes, ces riches ne sont pas tous des Bettencourt ou des Pinault, et leur patrimoine financier diversifié ne leur permet pas de contrôler un empire industriel, mais n'ont-ils pas un intérêt à la bonne marche des marchés financiers ? Plutôt que de parler d'une troisième classe, ne pourrait-on pas dire que le néolibéralisme a permis à la couche des cadres dirigeants, jusqu'alors strate supérieure du salariat, d'intégrer la classe des propriétaires du capital, ne serait-ce que sur un strapontin?

6 L'originalité de l'ouvrage réside aussi dans l'analyse des conséquences politiques des travaux pionniers de Stefania Vitali, James B. Glattfelder et Stefano Battiston (de l'Université de Zurich), concernant la structure de la propriété capitaliste au début du $\mathrm{XXI}^{\mathrm{e}}$ siècle $^{6}$. Ces auteurs ont utilisé de façon très innovante une gigantesque base statistique constituée par l'OCDE (Orbis 2007) pour retracer les liens capitalistiques entre 43000 sociétés transnationales à l'échelle mondiale. Duménil et Lévy rappellent leurs principaux résultats : 737 gros actionnaires (individus ou sociétés) contrôlent $80 \%$ du capital de ces sociétés dans le monde. Parmi les 50 plus importants, 45 sont des sociétés financières ; la moitié est états-unienne et 8 , britanniques ; 5 sont françaises et seulement 2, allemandes.

7 Ces institutions financières forment selon Duménil et Lévy un véritable gouvernement parallèle, le «centre économique institutionnel », qui est étroitement lié au «centre politique institutionnel » traditionnel par des relations informelles et de nombreuses passerelles ( revolving doors» dans le monde anglo-saxon ou "pantouflage » dans le 
contexte français). Cette alliance entre la haute fonction publique et la haute finance est l'un des piliers les plus solides du dispositif néolibéral, qui permet d'étouffer tout débat sur d'autres politiques possibles.

8 L'étude des économistes de Zurich montre donc que l'Europe continentale semble relativement à l'écart des réseaux financiers globalisés anglo-saxons qui dominent l'économie mondiale. Pour Duménil et Lévy, cela signifie que l'Europe et la France, audelà de leurs actuels choix néolibéraux, auraient encore la capacité de mener une autre politique économique. Une alliance entre les cadres et les classes populaires pourrait mettre en échec le néolibéralisme et constituer un « néomanagérialisme » industriel sous hégémonie des cadres, appuyé sur des contrôles de capitaux et un protectionnisme européen. Cependant, la France, davantage axée sur la finance que l'Allemagne industrialiste, aurait plus de difficultés à négocier cette «bifurcation » : l'État français apparaît plus favorable à l'industrie financière, plus «néolibéral» donc, que son homologue allemand davantage tenté par un cours «néomanagérial ».

9 Cette thèse peut sembler porteuse d'espoir. Cependant, elle ne semble pas vraiment confirmée par l'observation des stratégies des grands groupes européens, désormais alignés sur les logiques actionnariales. D'autres études statistiques, reposant sur des données peut-être plus récentes que celles d'Orbis 2007, aboutissent à des résultats sensiblement différents. Ainsi, entre 1999 et 2007, la part des investisseurs non résidents dans le capital des grandes entreprises allemandes est passée de $8 \%$ à $62 \%$ (et de $16 \%$ à «seulement » $49 \%$ pour le CAC $40^{8}$ ). Bien sûr, tous ces investisseurs «non résidents » ne sont pas anglo-saxons. Mais il semble bien que les liens traditionnels, de long terme, entre les grandes banques allemandes et les grands groupes industriels, aient été profondément remis en cause dans les années 2000, avec l'entrée très majoritaire de fonds d'investissement à la logique financière court-termiste au capital de ces groupes.

10 Au final, si les cadres dirigeants sont devenus des capitalistes, si les grands groupes européens semblent s'être alignés au cours de la dernière décennie sur des logiques purement financières, quel espace reste-t-il pour la bifurcation néomanagériale «à gauche » qu'appellent de leurs vœux les auteurs? Qu'est-ce qui pousserait les cadres à rompre leur lune de miel si fructueuse avec les détenteurs des capitaux pour s'allier avec les classes populaires? Les notations sur l'«aliénation» des cadres ou sur leur souffrance de devoir "faire le sale boulot", selon la formule de Christophe Dejours ${ }^{9}$ (p.177), sont certainement pertinentes pour les cadres de production, de vente, de gestion des ressources humaines, mais pourraient s'avérer insuffisamment mobilisatrices pour les cadres dirigeants et financiers (qui constituent, rappelons-le, la classe des cadres selon Duménil et Lévy).

11 Doit-on regretter le manque de plausibilité empirique de cette voie "néomanagériale »? Sans doute pas, car même si les auteurs se gardent bien de tout hymne inconsidéré à la croissance économique, il s'agirait probablement d'une impasse écologique. La réflexion sur la crise écologique et la nécessaire bifurcation des modèles productifs qu'elle implique n'occupent pas dans l'ouvrage toute la place qu'elles mériteraient. La «bifurcation» de Duménil et Lévy est pour l'essentiel un renversement des alliances de classes au sein d'un paradigme incontesté de l'efficience productive et de la technoscience ( $«$ il y a beaucoup à apprendre des voies industrialistes en matière d'efficience », p. 183). Cet hymne à l'industrie et à la technique ne risque-t-il pas de reproduire un imaginaire progressiste passablement 
daté ? Pourtant, nombre d'économistes posent désormais la question d'un changement complet de paradigme, vers une économie de la qualité et du bien-vivre, qui organiserait une décroissance systématique des consommations énergétiques et des productions matérielles, renonçant à l'idéal moderne de la maitrise rationnelle illimitée de la nature.

La réflexion stratégique que proposent Duménil et Lévy gagnerait aussi à s'inscrire dans une analyse sociologique et politique plus approfondie de la dynamique des luttes sociales dans la période néolibérale, et notamment depuis la crise de 2008. Après trente années de balkanisation du salariat, le syndicalisme et le mouvement ouvrier - la "gauche sociale ", comme l'appellent Duménil et Lévy - semblent avoir épuisé leurs capacités d'initiative et se replient sur des luttes défensives rarement victorieuses. La deuxième gauche qu'ils distinguent, la "gauche écologique ", semble osciller entre lobbying et action symbolique, sans grande prise sur le réel. La "gauche transformatrice immédiate " (l'économie sociale et solidaire et les mouvements de la «transition») se consacre à des alternatives locales sans projet global. Pour comprendre comment ces trois gauches pourraient peser réellement sur le cours des choses, il faudrait mener une analyse fine des multiples résistances à la grande vague de marchandisation que connaît la planète depuis trente ans, dans la lignée de la "grande transformation" de Karl Polanyi ${ }^{10}$, ouvrage auquel Duménil et Lévy se réfèrent implicitement dans leur titre sans jamais le citer.

On ne peut cependant pas leur faire le reproche de ne pas mener à bien cette tâche car même si des sociologues comme Michael Burawoy ${ }^{11}$ l'ont mise à l'ordre du jour, elle reste largement à accomplir, sans doute grâce à une démarche pluridisciplinaire à laquelle les travaux de Gérard Duménil et Dominique Lévy apporteraient à n'en pas douter un matériel très précieux.

\section{NOTES}

1. Burnham J. (1941), The managerial revolution. What is happening in the world, New York, The John Day company.

2. Galbraith J. K. (1967), The new industrial state, Boston, Houghton Mifflin.

3. Chandler A.D. (1977), The visible hand: the managerial revolution in American business, Cambridge, Mass., Belknap Press.

4. Bourdieu P. (1986), "The forms of capital ", in Richardson J. (ed.), Handbook of theory and research for the sociology of education, New York, Greenwood Press, pp. 241-258.

5. Institut national de la statistique et des études économiques.

6. Vitali S., Glattfelder J. B., Battiston S. (2011), "The network of global corporate control», Papers, $\mathrm{n}^{\circ}$ 1301.2363, arXiv.org. Disponible en ligne à l'adresse: http://arxiv.org/pdf/ 1107.5728.pdf ; consulté le 24 juin 2015.

7. Organisation de coopération et de développement économiques.

8. Lantenois C., Coriat B. (2009), «Montée des investisseurs institutionnels non résidents, mutations du corporate governance et stratégies d'entreprise. Évaluation et analyse à partir d'un panel de firmes françaises et allemandes (1999-2007) ", Document de travail du CEPN, n 2009-18. 
Disponible en ligne à l'adresse: http://www.univ-paris13.fr/cepn/IMG/pdf/wp2009_18.pdf ; consulté le 26 juin 2015.

9. Dejours C. (1998), Souffrance en France : la banalisation de l'injustice sociale, Paris, Le Seuil.

10. Polanyi K. (1944), The great transformation, New York, Toronto, Farrar \& Rinehart, inc.

11. Burawoy M. (2007), "Public sociology vs. the market», Socio-economic review, vol. $5, \mathrm{n}^{\circ} 2$, pp. 356-367.

\section{AUTEURS}

\section{THOMAS COUTROT}

Direction de l'animation de la recherche, des études et des statistiques (Dares). 